\title{
Composite fermions traversing a potential barrier
}

\author{
L. Brey ${ }^{1}$ and C. Tejedor ${ }^{2}$ \\ ${ }^{1}$ Instituto de Ciencia de Materiales (CSIC). Universidad Autónoma de Madrid C-12, Cantoblanco, 28049, Madrid, Spain. \\ ${ }^{2}$ Departamento de Fisica de la Materia Condensada. Universidad Autónoma de Madrid, Cantoblanco, 28049, Madrid, Spain.
} (October 10, 2018)

Using a composite fermion picture, we study the lateral transport between two two-dimensional electron gases, at filling factor $1 / 2$, separated by a potential barrier. In the mean field approximation, composite fermions far from the barrier do not feel a magnetic field while in the barrier region the effective magnetic field is different from zero. This produces a cutoff in the conductance when represented as a function of the thickness and height of the barrier. There is a range of barrier heights for which an incompressible liquid, at $\nu=1 / 3$, exists in the barrier region.

PACS number 73.40.Hm

The presence of a high magnetic field $B$ perpendicular to a two dimensional electron gas produces the Quantum Hall effect (QHE) characterized by the appearance of plateaus at rational values of $e^{2} / h$ of the off-diagonal conductance $\sigma_{x y}$ and zeros in the diagonal conductance $\sigma_{x x}$. QHE is usually analyzed separating the set of features in two, depending on whether the plateau appears at a filling factor $\nu_{e}=2 \pi \ell^{2} n$ which is an integer number (IQHE), or a fractional one (FQHE). In this expression $\ell=\sqrt{\hbar c / e B}$ is the magnetic length and $n$ the two dimensional electron density. The reason for this division is the different physical origin of the energy gap between occupied and unoccupied states of the system, which is responsible for the QHE. In the case of IQHE the gap originates from the quantization of the kinetic energy in the presence of $B$ while for the FQHE the gap is due to the electron-electron interaction. As from the experimental point of view both IQHE and FQHE present similar behavior, it is appealing to look for a unified representation of the two processes [1]. This is the goal of the composite fermions (CF) picture of the FQHE. In this framework, the relevant quasiparticles are $\mathrm{CF}$ built up as electrons attached to an even number, $\tilde{\phi}$, of flux quanta. Instead of working with the electronic Hamiltonian, a singular gauge transformation is performed and the system is described by a Hamiltonian for the CF which interact by both Coulomb and Chern-Simons terms [2]. Within a mean field approach, $\mathrm{CF}$ move in an effective magnetic field $B_{\text {eff }}=B-\tilde{\phi} n h c / e$. We made the choice of $\tilde{\phi}=2$ so that when $B$ is such that the electron filling factor is one half, $\mathrm{CF}$ do not feel any effective magnetic field and present a Fermi surface [3]. In the case of Coulomb interaction between the electrons, the Fermi surface does not dissapears when fluctuations are included [3,5]. At the same time, an incompressible electron liquid in the FQHE regime given by $\nu_{e}=p /(1+\tilde{\phi} p)$ corresponds to the IQHE regime for $\mathrm{CF}$ with filling factor $\nu_{C F}=|p|$ 梱田. In other words, a mean field aproach for $\mathrm{CF}$ gives an IQHE-like description for the FQHE although their physical origins are different. A strong support for the $\mathrm{CF}$ theory is that several predictions coming from it have been experimentally confirmed [6].

We study the transport of CF through a potential barrier in the presence of an external magnetic field, corresponding to an electronic filling factor $\nu_{e}=1 / 2$. As charge is expelled from the barrier region, the effective field $B_{\text {eff }}$ affecting the $\mathrm{CF}$ is not homogeneous anymore. This produces two effects: i) the localized effective field deflects the carriers in such a way that, if they do not have enough kinetic energy, they are not able to pass through the magnetic barrier and ii) a region of incompressible liquid can appear at the barrier. In particular we will show how an incompressible phase $\nu_{e}=1 / 3$, i.e. $\nu_{C F}=1$, forms and separates the two phases of compresible liquid $\nu_{e}=1 / 2$, i.e. CF with Fermi surface. This incompressible phase in the barrier region controls the current in the linear regime because it is stable in the presence of low density of impurities contrarily the magnetic barrier effect which is quenched by impurity scattering.

We consider that the potential barrier is along $x$ direction and the system is translationally invariant in the other one, $y$. Therefore we describe the effective magnetic field by using the Landau gauge $\mathbf{A}_{\text {eff }}=$ $\left(0, \int d x B_{\text {eff }}(x), 0\right)$. In order to compute conductances through the barrier, the required ingredient the probability that one CF coming from the left part of the barrier passes This must be computed taking into account the dependence of the effective magnetic field on the charge density, so selfconsistency between $B_{\text {eff }}(x)$ and $n(x)$ is required. This task is accomplished in the following way. We consider an external potential $V_{0}(x)$ which, after the charge rearrangement, will give a barrier for the $\mathrm{CF}$ of the form showed in Fig.1a; $V(x)=$ $V_{b}\left[\Theta\left(x+d_{b} / 2\right)-\Theta\left(x-d_{b} / 2\right)\right]$. For this given final potential it is necessary to know the amount of charge and the effective magnetic field in the barrier region. We obtain these quantities self-consistently in the Hartree approximation $[7]$. In this approach the Schrödinger equation for $\mathrm{CF}[7]$ takes the form,

$$
\begin{array}{r}
\left\{\frac { \hbar \omega _ { c } } { 2 } \ell ^ { 2 } \left[-\frac{d^{2}}{d x^{2}}+\right.\right. \\
\left.\left.\left(k_{y}+\frac{e}{c} \frac{A_{e f f}(x)}{\hbar}\right)^{2}\right]+V(x)\right\} \\
\frac{e^{i k_{y} y}}{L_{y}} \phi_{k_{y}, \varepsilon}(x)=\varepsilon \frac{e^{i k_{y} y}}{L_{y}} \phi_{k_{y}, \varepsilon}(x)
\end{array}
$$

here $\hbar k_{y}$ is the momentum of the $\mathrm{CF}$ in the $y$ direc- 
tion, $L_{y}$ is the sample length in the $y$-direction and $\omega_{c}=e B / m c$ is the cyclotron frequency, $m$ being the renormalized mass of the $\mathrm{CF}$. Note that in this expresion $V(x)$ is the final potential seen by the CF, i.e. the sum of the external potential, the Hartree potential and the potential induced by the motion of the magnetic flux tubes [7, 8]. The effective vector potential is

$$
A_{e f f}(x)=\frac{c \hbar}{e}\left(\frac{x}{\ell^{2}}-\tilde{\phi} \pi \int d x^{\prime} n\left(x^{\prime}\right) \operatorname{sgn}\left(x-x^{\prime}\right)\right)
$$

where the density $n(x)=\sum_{k_{y}, \varepsilon}\left|\phi_{k_{y}, \varepsilon}(x)\right|^{2} / L_{y}$ is a sum restricted to energies lower that the Fermi energy, which is $\hbar \omega_{c} / 2$ for the case of electronic filling factor $\nu_{e}=1 / 2$ [7]. By solving Eq.1-2, we get the self-consistent $n(x)$ and $B_{\text {eff }}(x)$. The amount of charge in the barrier region is obtained by integrating $n(x)$ in a region of width $D$. This is a cut-off, convenient for numerical calculations, large enough to contain all the effects coming from charge rearrangements. The amount of charge in the barrier region is represented by the average electron filling factor at the barrier $\nu_{e}^{b}$. It must be obviously smaller than $\nu_{e}=1 / 2$ and one can expect that the system tends to condensate in this region at values of the density where an incompressible liquid appears. We will see below that this is precisely the result we get.

Once we know the self-consistent effective magnetic field in the barrier region we are in condition to compute the transmission coefficient through the barrier. Since in the barrier region $B_{\text {eff }} \neq 0$, there is a step, $\Delta A=A_{\text {eff }}(-\infty)-A_{\text {eff }}(\infty)$, in the effective vector potential (see Fig.1(c)). This step produces that the $y$-component of the canonical momentum of the $\mathrm{CF}$ changes by $\frac{e}{c} \Delta A$, in passing from a point to the left of the barrier, $-D / 2$, to a point to the right, $D / 2$, which are not affected by the existence of the barrier. Therefore, in order to calculate the transmission coefficient for a CF with mechanical momentum $\hbar\left(k_{x}, k_{y}\right)$ and energy $\varepsilon=\hbar^{2}\left(k_{x}^{2}+k_{y}^{2}\right) / 2 m$, we match the two independent solutions of Eq.1 at energy $\varepsilon$, with the planewave $\left(e^{i k_{x} x}+\rho e^{-i k_{x} x}\right)$ at $x=-D / 2$ and with $\tau e^{i k_{x}^{\prime} x}$ at $x=D / 2$. Here $k_{x}^{\prime}=\left(k_{x}^{2}-\left(\frac{e}{c \hbar} \Delta A\right)^{2}+2 k_{y} \frac{e}{c \hbar} \Delta A\right)^{1 / 2}$, as obtained from the conservation of both $k_{y}$ and energy. From the matching of these wavefunctions the desired transmission coefficient $T\left(\varepsilon, k_{y}\right)=|\tau|^{2} k_{x}^{\prime} / k_{x}$ is obtained.

The first interesting property in the problem comes from the expression for $k_{x}^{\prime}$ : Only $\mathrm{CF}$ with incident mechanical momentum verifying the relation, $k_{x}^{2}-$ $\left(\frac{e}{c \hbar} \Delta A\right)^{2}+2 k_{y} \frac{e}{c \hbar} \Delta A>0$, can pass through the barrier. This restriction comes from the conservation of the mechanical momentum and the energy, and is very easily understood in classical terms. A charged incident particle with momentum $\mathbf{p}=\left(p_{x}, p_{y}\right)$, is deflected when arrives to a spatial region, $-D / 2<x<D / 2$, where a magnetic field $B$ exists. Only when the incident momentum is high enough, the particle is able to cross the finite $B$ region. Therefore, there is a region in momentum space, shown in Fig.1d, where the transmission coefficient through the barrier is zero. In the other regions in momentum space, the transmission coefficient can take finite values. This condition gives us a cutoff for the conductance of the $\mathrm{CF}$ system in the presence of a barrier.

The conductance is given by an integral of the transmission coefficients over all the incident states with energy $E_{F}$ [9],

$$
G=G_{0} \int_{-\pi / 2}^{\pi / 2} T\left(E_{F}, \sqrt{2 E_{F}} \sin \theta\right) \cos \theta d \theta,
$$

with $G_{0}=\frac{e^{2} k_{F}}{\hbar} L_{y}, k_{F}$ being the Fermi wavevector. This conductance for non-interacting $\mathrm{CF}$, treated in mean field approximation, corresponds to the conductance of the $2 \mathrm{D}$ electron gas at $\nu=1 / 2$ [3]. In the incident mechanical momentum space, states at the Fermi energy are on a semicircle centered at $(0,0)$ (see Fig.1d). If this semicircle is contained in the region of $T=0$ the conductance is zero. In order to have $G \neq 0$ the Fermi line must cross the boundary separating the region of $T=0$ from that of $T \neq 0$. This is only possible if the barrier is narrow or low enough to allow a large charge density in the barrier region, implying $B_{\text {eff }}$ is small there.

It must be stressed that, in the description we are using, the $\mathrm{CF}$ is always constituted by one electron carrying two flux quanta. Therefore, the scheme can not be directly applied to tunneling processes (i.e. $E_{F}<V_{b}$ ) in which the density at the barrier is practically zero. In such a case the $\mathrm{CF}$ at the barrier region could be strongly affected in its constitution.

The numerical results for $G$ and $\nu_{e}^{b}$ as a function of barrier height are presented in Fig.2 and Fig.3 for barriers thickness of $4 \ell$ and $16 \ell$, respectively. In order to see more clearly the effect of $B_{\text {eff }}$ on the $\mathrm{CF}$, the same magnitudes are plotted in dashed lines for electrons passing through a barrier without any magnetic field. In this case, both $G$ and $\nu_{e}^{b}$ decrease monotonously with incresing barrier height. When $V_{b}=E_{F}$ there is very small amount of charge at the barrier region and the conductance is enormously reduced because tunneling is the only mechanism for transport. The results are quite different for $\mathrm{CF}$, in the metallic regimen $\left(\nu_{e}=1 / 2\right)$, traversing a region with a potential barrier. The effect of the cutoff caused by $\Delta A$ on the transmission coefficient, produces the quenching of $G$ for barriers significantly lower than $E_{F}$. This is clear by noting that in the case of $\mathrm{CF}$ in the metallic regime, and supposing an abrupt magnetic barrier, the cutoff for $T \neq 0$ at $E_{F}$ is given by $\left(1-2 \nu_{e}^{b}\right) d_{b} / \ell=2 k_{F} \ell . \nu_{e}=1 / 2$ implies $k_{F}=\ell^{-1}$ [7] and we obtain that the critical $\nu_{e}^{b}$ for the barrier $4 \ell$ and $16 \ell$ thick are 0.25 and 0.469 respectively. From comparing Fig.2a (Fig.3a) with Fig.2b (Fig.3b), we see that the quenching of $G$ occurs roughly at these values of $\nu_{e}^{b}$.

The second, and even more important effect is observed in the electron filling factor at the barrier. This magnitude starts decreasing monotonously and remains finite even for barrier height at which $G=0$. But further 
increasing barriers, $\nu_{e}^{b}$ shows an abrupt decrease for a critical value of $V_{b}$. For $d_{b}=16 \ell$ a plateau with value $\nu_{e}^{b}=1 / 3$ develops before the abrupt decrease. The physical origin of this behaviour can be understood by looking at the schematic band structure of the system shown in Fig.4 for three increasing values of the barrier height and, consequently for increrasing effective magnetic field. The dispersion relation consists basically on two filled parabolas, with the bottoms shifted by the quantity $\frac{e}{c \hbar} \Delta A$. Each of these parabolas corresponds to $\mathrm{CF}$ located at the left and the right side of the barrier. Due to the existence of $B_{\text {eff }} \neq 0$ in the barrier region, some Landau-like bands (LLB) appears. For low barriers, i.e. low $B_{\text {eff }}$ and low $\Delta A$, the parabolas cross each other at low energy and the LLB are placed in the continuum defined by the parabolas and there are not localized states at the barrier region (Fig.4a). For increasing barrier, the shift between the bottom of the parabolas increases and a set of LLB appears in the region between parabolas (Fig.4b). When only the lowest energy LLB is occupied, an incompressible liquid, with $\nu_{C F}=1$, exists in the barrier region. Therefore the system is stable for $\nu_{e}^{b}=1 / 3$ giving the plateau observed in Fig.2 and Fig.3. As the LLB are dispersionless in the barrier region, the plateau at $\nu_{e}^{b}=1 / 3$ disappears suddenly when, for higher barriers, $B_{\text {eff }}$ is big enough so that the lowest energy LLB is empty and there are not localized occupied states at the barrier (Fig.4c). The plateau in the filling factor at the barrier is then the signature of the existence of an incompressible liquid at the barrier separating the two metallic regions at the left and the right.

An important issue is whether the two effects found here, the cutoff for $G$ and the incompressible region at the barrier, are robust with respect to the presence of a small random potential due to impurities or imperfections in the system. The existence of a random potential would relax the momentum conservation which is in the origin of the existence of the cutoff for $G$. Therefore the first consequence of having impurities is that the current would not be quenched as before. However the incompressible liquid is rather robust, with respect to the presence of a weak random potential, provided that the energy gap in the barrier region persists in the presence of impurities. Therefore, we expect that the imcompressible region would persist in samples in which the mobility is high enough so that the $\nu_{e}=1 / 3 \mathrm{FQHE}$ can be observed. The existence of an incompressible liquid in the barrier region (no states at $E_{F}$ ) produces that $G$ would be zero for barriers in which $\nu_{e}^{b}$ presents a plateau. Therefore, the quenching of the conductance persist although for barriers higher than before and due to a different physical origin.

We are indebted to L.Martin-Moreno for fruitful discussions. The help received from J.J. Dorado is deeply acknowledged. This work has been supported in part by the Comisión Interministerial de Ciencia y Tecnologia of Spain under contract No. MAT 94-0982-C02.
[1] J.K.Jain, Phys. Rev. Lett. 63, 199 (1989); Adv. Phys. 41, 105 (1992).

[2] A.López and E.Fradkin, Phys.Rev.B 44, 5246, (1991).

[3] B.I.Halperin, P.A. Lee and N. Read, Phys.Rev. B 47, 7312 (1993).

[4] Y.B.Kim et al., Phys. Rev. B 50, 17917 (1994).

[5] We treat $\mathrm{CF}$ as having a renormalized finite effective mass although this is still a matter of controversy and such a mass has not been measured for $\nu_{e}=1 / 2$.6.

[6] R.L.Willet et al., Phys.Rev.Lett. 71, 3846 (1993); W.Kang, et al., ibid. 71, 3850 (1993); R.R.Du et al., ibid. 70, 2944 (1993); D.R.Leadley et al., ibid 72, 1906 (1994); R.R.Du et al., Solid State Comm. 90, 71 (1994); V.J.Goldman et al., Phys.Rev.Lett. 72, 2065 (1994); H.C.Manoharan et al., Phys. Rev. Lett. 73, 3270 (1994); R.R.Du et al., Phys. Rev. Lett. 73, 3274 (1994).

[7] Luis Brey, Phys. Rev. B 50, 11861 (1994).

[8] C.B. Hanna, R.B. Laughlin and A.L. Fetter, Phys.Rev. B 40, 8745 (1989); A.L.Fetter and C.B. Hanna, Phys.Rev. B 45, 2335 (1992); Q.Dai et al., Phys.Rev. B, 46, 5642 (1992).

[9] F.M.Peeters and A.Matulis, Phys. Rev. B 48, 15166 (1993); A.Matulis et al., Phys. Rev. Lett. 72, 1518 (1994).

FIG. 1. Schematic plot of (a) the potential barrier $V(x)$, (b) charge density profile $n(x)$, and (c) effective vector potential $A_{\text {eff }}(x)$. In (d), the separation between the regions with $T=0$ and with $T \neq 0$ is plotted, in the space of the mechanical momentum of the incident CF. The semicircles are the Fermi lines in the case in which $G=0$ (dashed line) and in the case in which $G$ can be different from zero (dotted line).

FIG. 2. Variation of the filling factor in the barrier region, $\nu_{e}^{b}$ (a) and the conductance $G(\mathrm{~b})$, as a function of the barrier height $V_{b}$ in units of $\hbar \omega_{c}$, for a barrier $4 \ell$ thick. The dashed lines correspond to the case of electrons without magnetic field.

FIG. 3. Variation of the filling factor in the barrier region, $\nu_{e}^{b}$ (a) and the conductance $G(\mathrm{~b})$, as a function of the barrier height $V_{b}$ in units of $\hbar \omega_{c}$, for a barrier $16 \ell$ thick. The dashed lines correspond to the case of electrons without magnetic field.

FIG. 4. Schematic representation of the band structure of $\mathrm{CF}$ in the metallic regime and in presence of a potential barrier for different heights of the potential barrier. 